\title{
Visual beats: Preliminary observations of perceived rate as a function of retinal locus stimulated'
}

\author{
RATHE KARRER ${ }^{2}$ AND JOHS CLAUSEN \\ ILLINOIS STATE PEDIATRIC INSTITUTE AND INSTITUTE FOR BASIC RESEARCH IN MENTAL RETARDATION
}

Visual beats produced by simultaneous presentation of two different flicker frequencies were used to determine the temporal processing of various retinal areas. The dichoptic presentation of two different flicker frequencies to the center and periphery of the retina resulted in a perceived beat rate that equalled the physical beat frequency. When the stimuli were presented to more discrete retinal areas (temporal vs temporal and nasal vs temporal), the perceived beat rate also equalled the physical beat frequency. The data indicate that different flicker frequencies stimulating divergent retinal loci can be represented accurately and simultaneously in the visual system: Differences in temporal resolution at the different retinal loci do not occur for this repetitive stimulation.

Differences in temporal processing for stimuli presented to the peripheral and foveal retina are often reported (e.g., flicker in lateral vision appears slower than in central vision, Le Grand, 1957). It is well known that under certain conditions the periphery is more sensitive to flicker. Lichtenstein et al (1963) found no difference between peripheral and foveal stimulation for number of flashes counted, but did find a difference between these two areas for perceived flicker rate. It is difficult to understand why the perceived rate should look slower in the periphery while the number of flashes counted remains the same. There is also evidence that visual latencies differ with distance from the fovea (Lichtenstein \& White, 1961) as well as with peripheral (nasal vs temporal) locus stimulated (Rutschmann, 1966).

It is possible to study temporal processing by the visual system with the use of a beat technique. The perceived rate of visual beats arising from two different flicker frequencies in full-field dichoptic presentation indicates accurate following at rates as high as 80 pulses per second (Karrer, 1967). A beat frequency that equals the difference between the primary flicker frequencies (indicating accurate following of the flicker) can also be seen from dim flicker in the region of scotopic vision. This problem is further explored in the following preliminary studies. If the processing time of the peripheral retina is different than the fovea, the perceived beat rate should change as a function of retinal areas stimulated.

\section{Method \\ EXPERIMENT 1}

Flicker produced by two General Radio Strobotacs (Model 1531A) was presented in dichoptic view. The brightness of these sources was $400 \mathrm{~mL}$ measured with a MacBeth Illuminometer at fusion of 85 pulses per second (pps).

The flicker source to the left eye was in direct frontal view behind a ground glass screen 1-2 in. from the eye. This resulted in the flicker filling all but the extreme peripheral visual field. The right eye flicker source was located at a $90 \mathrm{deg}$ angle to the $S$, behind a similar screen also 1-2 in. from the eye. When the $S$ was in position and fixated a spot directly ahead, the flicker in the right eye appeared at the extreme right edge of the field (from $80 \mathrm{deg}$ to the limit of vision). Neither eye could see the contralateral stimulus. Observations were also obtained for dichoptic frontal-frontal view of the stimuli (see Karrer, 1965, 1967, for further details of apparatus and procedures).
The S's task was to look for and tap in synchrony with the perceived beats. These taps and the physical beat frequency (Bf) were recorded on a polygraph. The $E$ monitored eye fixation by watching the S's right eye and disqualified any data where fixation was not maintained. Three pairs of flicker frequencies (F1-F2) of 10 vs 11 pps, 20 vs 21 pps, and 35 vs 36 pps were presented in random order in a session (30-45 min). Sessions were repeated on three separate days. The frequencies were randomly adjusted so as to give beat frequencies ranging anywhere from 0.5 to 1.5 beats per second (bps), e.g., 10.6 vs $11.2=0.6 \mathrm{bps}$, or 9.7 vs $11.1=1.4$ bps. All series of more than five successive taps were scored. The number of samples per session for each frequency varied as a function of the S's motivation to cooperate. Total observations per frequency for each $S$ ranged from 6 to 16 . Beat frequency and perceived beat rate $(\mathrm{Br})$ were determined from the polygraph records to the nearest $0.5 \mathrm{~mm}(0.02 \mathrm{sec})$ and converted into the mean ratios $\mathrm{Bf} / \mathrm{Br}$. Four adult male experienced observers (including the authors) served as Ss.

\section{Results and Discussion}

For each stimulus pair all Ss reported beats which appeared primarily as a rhythmic change in flicker rate and lateral movement in the less bright right visual field. If the $\mathrm{S}$ attended to the right peripheral flicker while maintaining fixation, an apparent slight spreading of the diffuse flicker toward the center of the field rhythmically occurred.

Table 1 presents each $\mathrm{S}$ 's mean ratio $\mathrm{Bf} / \mathrm{Br}$ for each condition. Analogous data for frontal-frontal presentation are provided in the parentheses. Since the ratios are very close to unity, it is obvious that the beat is seen at a rate equal to the beat frequency. The slightly greater divergence from unity for the peripheral condition is best attributed to the difficulty in perceiving these beats. Thus, the stimulus frequencies are accurately represented in the visual system whether they fall on large areas of the center or periphery of the retina.

\section{EXPERIMENT 2}

By presenting the stimuli to restricted peripheral retinal areas (nasal and temporal), further evidence of the role of retinal locus in beat perception was obtained. With this type of presentation it is possible to vary the site of interaction in the retina and the brain of the two stimulus frequencies.

\section{Methods and Procedure}

Two Plexiglas rods ( 0.25 in. OD) serving as light guides were attached to the center of the two flicker sources (Grass

Table 1

Ratios of Beat Frequency to Perceived Beat Rate (Bf/Br) for Flicker in Peripheral vs Frontal View (values in parentheses are for frontal vs frontal view)

F1-F2 Frequency Combinations (pps)

\begin{tabular}{ccrr} 
Subject & $10-11$ & \multicolumn{1}{c}{$20-21$} & \multicolumn{1}{c}{$35-36$} \\
\hline 11 & $.99(1.00)$ & $.95(.98)$ & $1.04(1.00)$ \\
16 & $1.04(.99)$ & $1.01(.98)$ & $1.05(1.00)$ \\
21 & $1.03(1.01)$ & $.97(1.01)$ & $1.10(1.02)$ \\
23 & $.90(.97)$ & $1.02(.99)$ & $.90(.99)$ \\
\hline
\end{tabular}


Table 2

Mean Ratios of Beat Frequency to Perceived Beat Rate $(\mathbf{B f} / \mathbf{B r})$ for the Different Loci of Stimulation ( $\mathrm{N}=$ number of $30 \mathrm{sec}$ tapping series)

\begin{tabular}{ccc}
\hline & $\begin{array}{l}\text { Temp-Temp. } \\
\text { (dichoptic) }\end{array}$ & $\begin{array}{c}\text { Nasal-Temp. } \\
\text { (monocular) }\end{array}$ \\
\hline S 1 & $0.99 \pm 0.04$ & $0.99 \pm 0.04$ \\
$\mathrm{~N}=23$ & $\mathrm{~N}=7$ \\
S 2 & $1.00 \pm 0.03$ & $1.04 \pm 0.05$ \\
& $\mathrm{~N}=38$ & $\mathrm{~N}=6$ \\
\hline Total & $1.00 \pm 0.02$ & $1.02 \pm 0.03$ \\
& $\mathrm{~N}=61$ & $\mathrm{~N}=13$ \\
\hline
\end{tabular}

Photostimulators, Model PS2). The surrounding lamp area and the lengths of rod were masked with black paper and tape so that flicker was visible only at the distal end of each rod. The rods supplied a small $3.5 \mathrm{deg}$ spot of flicker 2 in. from the eye. They were appropriately placed so as to stimulate the temporal loci of each eye (dichoptic), or the nasal and temporal loci in the same eye (monocular). The S placing his chin on a rest, fixated a small red spot in the center of the field. The stimuli were further spatially isolated by placing a thin piece of black paper between them which projected outward from the facial contour. Positioning of the rods was precise enough so that fixation of the spot was necessary for simultaneous visibility of both stimuli.

It was not possible to measure the brightness of the dim flicker at the end of the rods. The room illumination was $0.01 \mathrm{~mL}$ and Ss were dark adapted for $10 \mathrm{~min}$ before obser:ing. Observations were obtained for the F1-F2 frequency pairs $15-16 \mathrm{pps}$ and 19-20 pps. Beat frequencies were randomly varied between 0.6 and 1.6 bps to control for a tapping rate set. The Ss looked for beats and tapped in synchrony for a 30-sec period if beats were perceived. At least six series of taps were counted for each $\mathrm{S}$ at each of the two conditions. Again, the ratio $\mathrm{Bf} / \mathrm{Br}$ was determined for each series, and the mean ratios for each $S$ computed for each condition. The two authors served as Ss.

\section{Results and Discussion}

For the two Ss, the mean ratios $\mathrm{Bf} / \mathrm{Br}$, and standard errors of the means at each condition are shown in Table 2.

The perceived beat rate again equals the beat frequency. The visual processes serving the different retinal loci accurately and equally follow the stimulus frequencies. Although the Ss tried to determine if they saw the beat in their contralateral eye during monocular stimulation, they were unable to decide which eye gave the beat. The appearance of the beat was similar to that of the peripheral-frontal beats of Experiment 1, except that the beat was very weak and much less area was involved. If, while maintaining the tedious fixation, the $\mathrm{S}$ attended to one of the rod ends, the beat appeared as a rhythmical and very faint change in flicker rate. It was generally easier to attend to the space between the two areas of flicker where a vague lateral to-and-fro movement appeared.

The beat for the monocular nasal-temporal condition was easier to see than that during dichoptic temporal-temporal stimulation but more difficult to perceive than that during dichoptic nasal-temporal stimulation. ${ }^{3}$ Interaction at the retinal level in the monocular nasal-temporal condition may be responsible for a stronger beat than the temporal-temporal condition. Of particular interest is the temporal-temporal beat since there is only limited interaction between the noncorresponding points of the two hemispheres. On the assumption that the perceived beat might be spurious, all alignments of the stimuli were double-checked and additional observations were obtained (hence the larger $\mathrm{N}$ for this condition). The Ss remained consistent in their beat perception and could discriminate beat presence from beat absence. The still greater effectiveness of the dichoptic nasal-temporal stimulation in producing beats might indicate that frequency registration and interaction (i.e., beat perception) is more a function of central neural processes. The perception of beats during the dichoptic temporal-temporal condition supports this interpretation.

Entoptic stray light, by spreading the area of stimulation, may contribute to or even be responsible for the beat correspondence of the different loci. It may be that there is enough stray light to stimulate homologous areas resulting in equal temporal processing and, therefore, a beat ratio of unity. The fact that dichoptic nasal-temporal beats are easier to see than monocular nasal-temporal beats is evidence against this interpretation. Also, there is no reason to believe that stray light should not have acted similarly in the previous studies (cf. Le Grand, 1957) of visual processing which found differences between the loci. It seems to follow that stray light is probably too weak to play a role in processing of short repetitive pulses.

These studies demonstrate that different flicker frequencies presented simultaneously in peripheral or frontal view and in specific retinal loci can be represented accurately and simultaneously in the visual system. Differences in temporal resolution of light pulses at different retinal loci do not occur in the present results. These studies and others (Karrer, in press) also imply a sizable amount of central interaction between the two eyes. It is of interest to further pursue the presence and perceived rate of dichoptic beats in order to more fully understand their basis as well as the apparent discrepancy between beat technique data and previous data using different methods. Studies utilizing more precise techniques are planned to extend the present results.

\section{REFERENCES}

KARRER, R. Visual beat phenomena and their relation to the temporal characteristics of perception. Unpublished Ph.D. dissertation, New School for Social Research, 1965.

KARRER, R. Visual beat phenomena as an index to the temporal characteristics of perception. Journal of Experimental Psychology, 1967, 75, 372-378.

KARRER, R. Visual beats: Differential brightness of the stimuli and estimations of brightness. Vision Research, in press.

Le GRAND, Y. Light, color, and vision. London: Chapman \& Hall, 1957.

LICHTENSTEIN, M., \& WHITE, C. T. Relative visual latency as a function of retinal locus. Journal of the Optical Society of America, 1961, 51, 1033-1034.

LICHTENSTEIN, M., WHITE, C. T., SIEGFRIED, J. B., \& HARTER, M. R. Apparent rate of flicker at various retinal loci and number of perceived flashes per unit time: A paradox. Perceptual \& Motor Skills, $1963,17,523-536$.

RUTSCHMANN, R. Perception of temporal order and relative visual latency. Science, 1966, 152, 1099-1101.

\section{NOTES}

1. Taken in part from a Ph.D. dissertation submitted by the first author to the New School for Social Research, 1965. We thank Howard Gruber for his generous guidance and Herb Kohn for suggestions on the manuscript.

2. Address: Illinois State Pediatric Institute, Chicago, Illinois 60608.

3. A dichoptic condition of nasal vs temporal loci giving an interaction site in one hemisphere was also used. Unfortunately, during analysis of the data, an error in setting the stimulus frequencies was discovered which rendered most of the data invalid. Three observations by one of the observers were usable and the ratios $\mathrm{Bf} / \mathrm{Br}(0.85,0.88,0.94)$ indicated good following.

(Accepted for publication October 7, 1968.) 